\title{
THE MOTION OF MINERAL SAND PARTICLES ON THE ROLL IN HIGH TENSION SEPARATORS
}

\author{
D. EDWARD, P.N. HOLTHAM AND T. KOJOVIC \\ Julius Kruttschnitt Mineral Research Centre, The University of \\ Queensland, Isles Rd., Indooroopilly, Qld. 4068, Australia
}

\begin{abstract}
A number of models of the high tension roll separator have been described in the literature. All the models, in their calculation of the centrifugal force component of the force balance, appear to assume that particles introduced onto the top of the roll instantaneously reach roll speed. This paper questions that assumption. A theoretical analysis of the forces involved suggests that particles almost certainly do not reach roll speed (even if they have been charged in a corona field) until they have been carried a considerable distance with the roll. Experimental evidence was found to confirm the theoretical analysis, with $150-180 \mu \mathrm{m}$ zircon particles having a speed of $180 \mathrm{rpm}$ after travelling 55 degrees on a roll revolving at $275 \mathrm{rpm}$.
\end{abstract}

(Received July 25, 1994, accepted August 22, 1994)

\section{INTRODUCTION}

Mineral sands represent an important product of the Australian mining industry. Rutile, leucoxene, ilmenite, zircon and monazite are recovered along the coast of Western Australia, from Geraldton in the north to Capel in the south, and on the east coast between Newcastle (New South Wales) and Brisbane (Queensland).

The separation of mineral sands generally occurs in two stages. The first stage is wet gravity separation which rejects the low density minerals, such as quartz, which comprise up to $90 \%$ of the material mined. The second stage of concentration is dry separation, and is used to upgrade the concentrate from the 
gravity stage into separate mineral products. The majority of dry plant separation is carried out by electrostatic and magnetic separators, and a secondary wet gravity stage rejects the remaining low density material. The principal electrical separation units are the high tension roll (HTR) and electrostatic plate (ESP) machines.

A considerable literature devoted to the understanding and modelling of HTR separation has developed over some three decades (for example Mora [1], Nilkuha [2], Morrison [3] and Dance [4]). The most recent work has resulted in phenomenological models for both HTR and ESP machines which have been incorporated into a PC-based simulation package - JKSimSand [4]. This software allows separation in an HTR machine to be optimised for a given mineral assemblage on a size by mineral basis. The effect of machine variables such as voltage, electrode position, roll speed and splitter positions can be rapidly evaluated, and the model also allows the effect of machine ambient conditions such as local relative humidity and sand temperature to be explored [5].

However, the model, while based on the physics of the process includes a number of empirical constants which have to be determined by fitting or calibration. It is the aim of the electrostatic separator research programme at the JKMRC to reduce the degree of empiricism of the model in order to enhance its robustness and utility to the industry as a predictive tool for process optimisation. This paper describes a part of the investigation concerned with particle motion on the HTR roll.

\section{MODELLING HTR SEPARATOR}

In principle, HTR modelling appears straightforward. Each particle on the roll is subject to a number of forces, varying in magnitude with time and position on the roll. The magnitude of these forces determines how the particle behaves, and hence its separation response. The major radial forces are gravity $\mathrm{F}_{\mathrm{GR}}$, centrifugal $\mathrm{F}_{\mathbf{C}}$, electrostatic pinning $\mathrm{F}_{\mathrm{P}}$ and electrostatic image attraction $\mathrm{F}_{\mathrm{I}}[4]$. These are shown diagrammatically in Figure 1. 


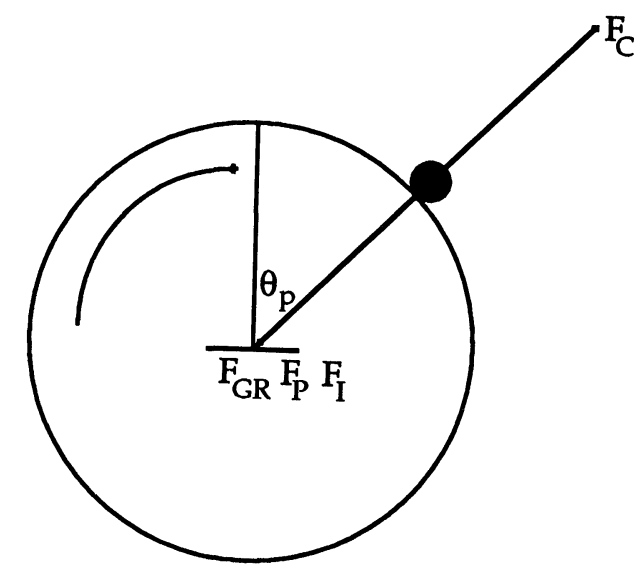

Fig.1 Radial forces acting on a particle on the HTR roll

The image force is the electrostatic force between the particle and the image charge it induces on the grounded roll. The pinning force is the electrostatic force acting on the charged particle in the presence of the static electric field. The radial force balance is then:

$$
F_{C}=F_{G R}+F_{I}+F_{P}
$$

The forces in eq. (1) may, in principle, be calculated separately using elementary physics, and the theoretical point of departure from the roll for an isolated particle can then be determined. For spherical particles, the forces are given by:

$$
\begin{aligned}
& F_{C}=\frac{\pi}{6} d^{3} \rho \omega_{r^{2}} R \\
& F_{G R}=\frac{\pi}{6} d^{3} \rho g \cos (\theta) \\
& F_{I}=\frac{1}{4 \pi \varepsilon_{0}} \frac{q^{2}}{d^{2}} \\
& F_{P}=q E
\end{aligned}
$$


where $d$ is the particle diameter, $\rho$ the mineral density, $\omega_{\mathrm{r}}$ the roll speed, $R$ the roll diameter, $\theta$ the angular position on roll, $\epsilon_{0}$ the permittivity of free space, $q$ the instantaneous particle charge and $E$ the static electric field strength.

The force balance defined by eqs. (1) to (5) implicitly assumes that the particle and roll surface are travelling at the same velocity $\omega_{\mathrm{r}}$, and this assumption has been widely adopted (for example [3], [6], [4]). However, if the particles are travelling at a velocity other than $\omega_{r}$, calculation of the centrifugal force using eq. (2) may be significantly in error.

Dance [4] in evaluating the radial force balance given by eq. (1), found it necessary to scale the expression for centrifugal force by a large factor $(0.125)$ in order to obtain satisfactory agreement between experimental and predicted results for the angle of departure of particle from the roll.

The general consensus from the literature is that no significant forces have been omitted from eq. (1), which suggests that the assumption that particle velocity almost instantaneously reaches roll velocity may not be valid. Thus the objective of the work described below was to investigate the assumption that particle speed equals roll speed both theoretically and experimentally, with the aim of eliminating the centrifugal force correction factor in the JKSimSand HTR model.

\section{PARTICLE MOTION ON A ROTATING ROLL}

\section{The Mechanical Forces}

The first analysis of the mechanical forces acting on a particle in a motion on a roll rotating with angular velocity $\omega_{\mathrm{r}}$ appears to have been carried out by Cakir et al. [7]. They found that particle motion could be described by four cases or regions:

1. particle accelerating up to roll speed due to frictional forces, particle angular velocity $\omega_{\mathrm{p}}<\omega_{\mathrm{r}}$

2. particle travelling at roll speed, $\omega_{\mathrm{p}}=\omega_{\mathrm{r}}$

3. particle accelerating forward to a speed greater than roll speed, $\omega_{\mathrm{p}}>\omega_{\mathrm{r}}$ 
4. particle leaving roll due to centrifugal force and following a ballistic trajectory, $\omega_{\mathrm{p}}>\omega_{\mathrm{pmax}}$.

In the absence of electrical or magnetic forces, there are three forces acting on a particle (Figure 2): the gravitational force $\mathrm{F}_{\mathrm{G}}$ acting vertically downwards, which can be resolved into tangential and radial components $F_{G T}$ and $F_{G R}$; the centrifugal force $F_{C}$ acting radially to the roll; and the frictional force $F_{F}$ proportional to the net radial force, which acts tangentially to the roll in the direction shown in Fig. 2, when the particle speed is less than the roll speed.

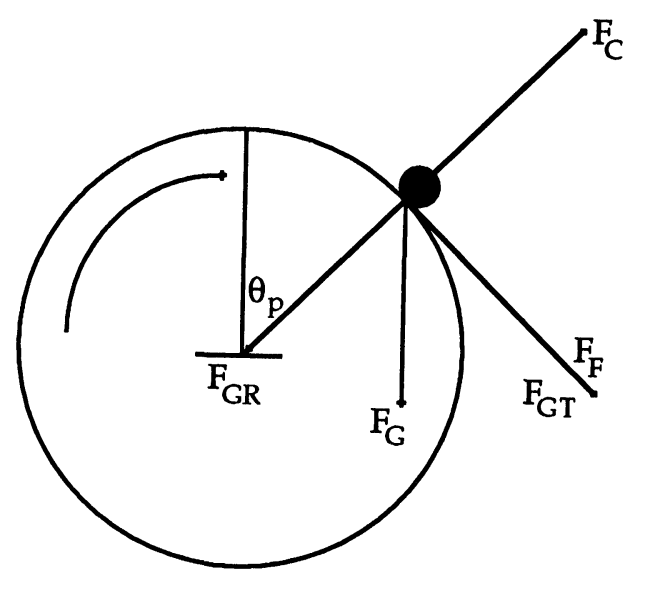

Fig. 2 Mechanical forces acting on a particle at angle $\theta_{\mathrm{p}}\left(\omega_{\mathrm{p}}<\omega_{\mathrm{r}}\right)$

It was assumed by Cakir et al. [7] that the particles were sliding not rolling and the coefficient of dynamic friction $\mu_{\mathrm{d}}$ was used in their analysis, except for region 2 where the static friction coefficient $\mu_{\mathrm{s}}$ applies. It will be shown later that experimental evidence suggests the particle are in fact rolling. In any analysis this can probably be accounted for by lowering the coefficient of friction.

The tangential force balance on a particle is:

$$
\mathrm{F}_{\mathrm{T}}=\mathrm{F}_{\mathrm{F}}+\mathrm{F}_{\mathrm{GT}}
$$


where $F_{T}$ is the total tangential force acting to accelerate the particle up to roll speed.

When the particle is in region 1 , i.e. $\omega_{\mathrm{p}}<\omega_{\mathrm{r}}$, then

$$
F_{F}=\mu_{d}(\text { nett radial force })=\mu_{d} m\left(g \cos \theta_{p}-\omega_{p}^{2} R\right)
$$

and hence

$$
\mathrm{F}_{\mathrm{T}}=\mu_{\mathrm{d}} \mathrm{m}\left(\mathrm{g} \cos \theta_{\mathrm{p}}-\omega_{\mathrm{p}}{ }^{2} \mathrm{R}\right)+\mathrm{mg} \sin \theta_{\mathrm{p}}
$$

where $m$ is the particle mass, $R$ the roll diameter and $\theta_{\mathrm{p}}$ the angle of the particle on the roll from the vertical. The tangential acceleration $a_{T}$ of the particle is given by:

$$
\begin{aligned}
& a_{T}=F_{T} / m=\left(F_{F}+F_{G T}\right) / m \\
& a_{T}=\mu_{d}\left(g \cos \theta_{p}-\omega_{p}^{2} R\right)+g \sin \theta_{p}
\end{aligned}
$$

Note that the acceleration is independent of particle mass and size. The first region ends and the second region begins when the particle speed equals the roll speed, i.e. $\omega_{\mathrm{p}}=\omega_{\mathrm{r}}$, and the tangential acceleration $a_{T}$ is zero. In this region the frictional force $F_{F}$ remains greater than the tangential component of the gravity force $F_{G T}$ but now acts in the opposite direction to that shown in Fig. 2. Eq. (6) can then be re-arranged to:

$$
\omega_{\mathrm{p}}{ }^{2}=\frac{\mathrm{g}}{\mu_{\mathrm{s}} \mathrm{R}}\left(\mu_{\mathrm{s}} \cos \theta_{\mathrm{p}}+\sin \theta_{\mathrm{p}}\right)
$$

where $\mu_{\mathbf{s}}$ is now the static friction coefficient. Depending on the roll speed, region 2 may or may not exist.

The third region applies when the frictional force is exceeded by the tangential component of the gravitational force. The particle begins to slip forward on the roll, accelerating once more until the point is reached at which the centrifugal force $F_{C}$ exceeds the radial component of the gravitational force $F_{G R}$ and the particle leaves the roll. The total tangential force in this region is now given by: 


$$
\mathrm{F}_{\mathrm{T}}=\mathrm{F}_{\mathrm{GT}}-\mathrm{F}_{\mathrm{F}}
$$

as before $a_{T}=F_{T} / m$, hence

$$
\mathrm{a}_{\mathrm{T}}=\mathrm{g} \sin \theta_{\mathrm{p}}-\mu_{\mathrm{d}}\left(\mathrm{g} \cos \theta_{\mathrm{p}}-\omega_{\mathrm{p}}{ }^{2} \mathrm{R}\right)
$$

Cakir et al. [7] solved eqs. (6), (7) and (8) analytically for various values of friction coefficient $\mu$ to obtain the angular velocity of a particle as it was carried around the rotating roll. Their solution has been re-worked more compactly and with typographical errors corrected by Stradling [8].

In the present work the equations were solved numerically in order to check the quite complex analytical solutions of [7], and also to allow rapid investigation of changes of variables such as roll speed, friction coefficient etc. Numerical solution of the equations was carried out in two ways: firstly, using a personal computer spreadsheet package and secondly a purpose-written program. In both cases, the radial and tangential force balances were evaluated iteratively for small angular increments around the roll, allowing the particle acceleration and angular velocity to be successively calculated at at increment.

\section{Verification of the Numerical Solutions}

The numerical solutions were tested for correctness and sensitivity to the angular increment using the case of a frictionless particle placed on top of a smooth stationary roll, for which a simple analytical solution is available. If the particle is slightly disturbed from its initial position allowing it to slide down the roll, the angle from the vertical at which it leaves the roll can be shown to be $\cos ^{-1}(2 / 3)$ degrees. An angular increment of 0.001 degrees was found to give an acceptable trade-off between speed of computation and numerical accuracy.

The numerical solutions were then tested with the conditions used by Cakir et al. [7]; a roll diameter of $0.152 \mathrm{~m}$, friction factors of 0.1 and 0.4 and with particles placed on the roll with zero tangential velocity at the maximum angle before top dead centre possible at which they would still be carried around by frictional forces. The results are shown in Fig. 3. Comparison with Figs. 4 and 5 which use the analytical solution of [7], and in which their format is adopted, shows good 
agreement. Using their nomenclature, point $X$ identifies the end of region 2, and point $Y$ the point where particles leave the roll (the end of region 3 ).

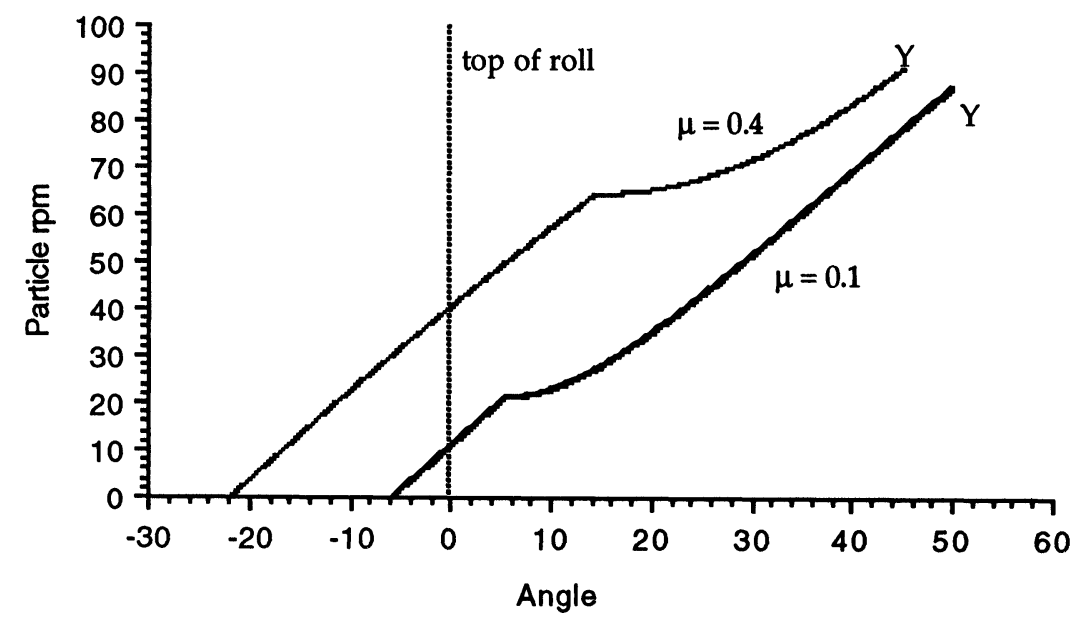

Fig. 3 Particle speed as a function of position on the roll numerical solution (roll diameter $0.152 \mathrm{~m}$ )

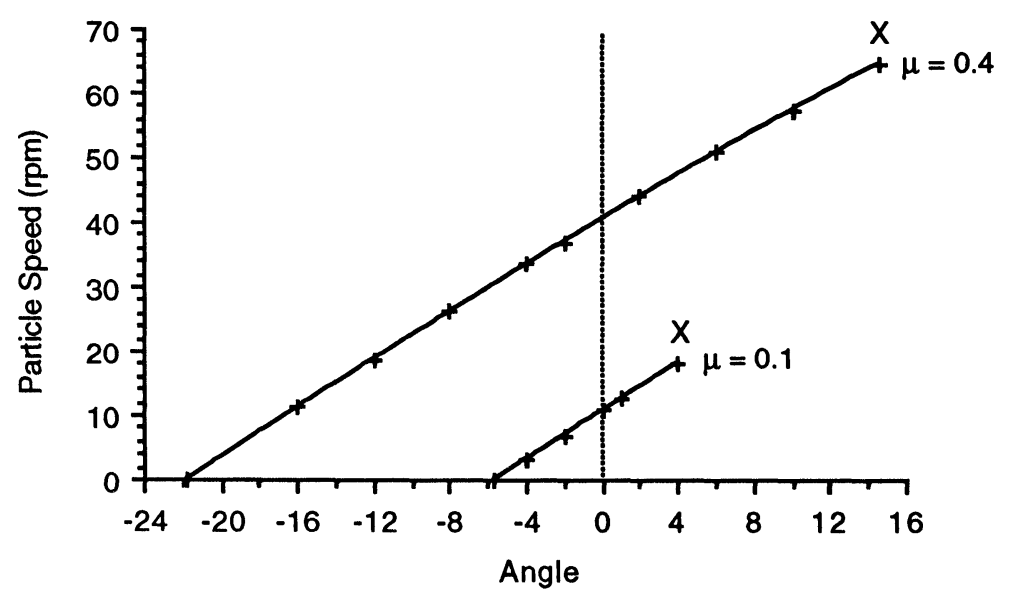

Fig. 4 Particle speed as a function of position on the roll analytical solution for regions 1 and 2 (roll diameter $0.152 \mathrm{~m}$ )

It can be seen from Figures 3 to 5 that for industrial roll speeds (typically 200 to $250 \mathrm{rpm}$ ) region 2 will not exist, as particles will start to accelerate under gravitational forces before reaching roll speed. It is also clear that particles not 
subject to additional inwardly acting radial forces will leave the roll well before reaching these roll speeds.

Electrical forces due to corona charging of particles on the roll will, however, increase the net radial force acting on the particles, allowing them to accelerate to greater angular velocities. The effect of including these forces is considered in the next section.

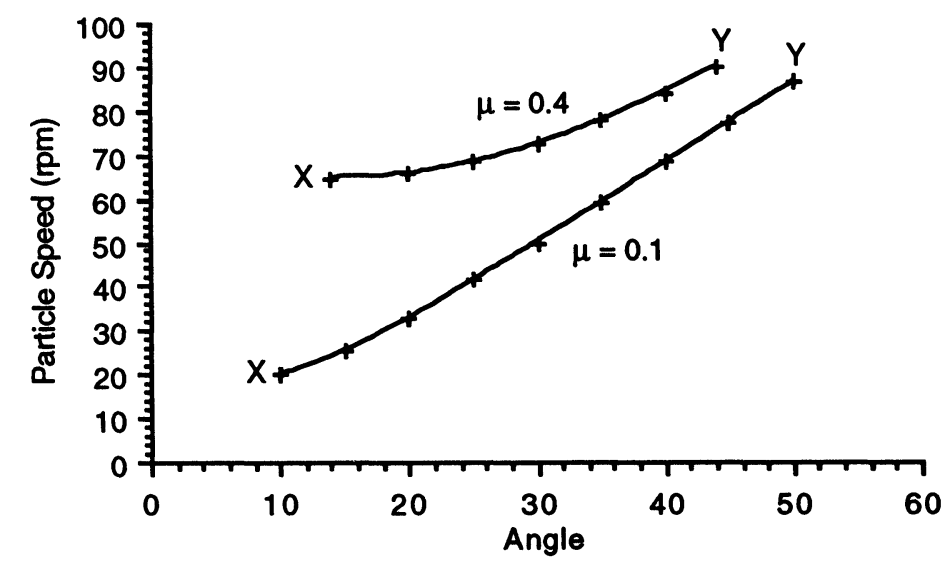

Fig. 5 Particle speed as a function of position on the roll analytical solution for region 3 (roll diameter $0.152 \mathrm{~m}$ )

\section{The Effects of Corona Charging of Particles}

If the particles on the roll are now charged by a corona electrode, the radial forces are augmented by the electrostatic and image forces introduced earlier. These forces vary with particle position on the roll. Initially, both are zero but as as the particle enters the corona field and is rapidly charged up to some maximum value, both forces reach a maximum value which is maintained until the particle is carried out of the corona field.

The charge on the particle then begins to decay, rapidly for conductors such as ilmenite and slowly for non-conductors such as zircon, with a corresponding decrease in the electrostatic and image forces. This process is illustrated diagrammatically in Fig. 6a, for moderately conducting particle. 


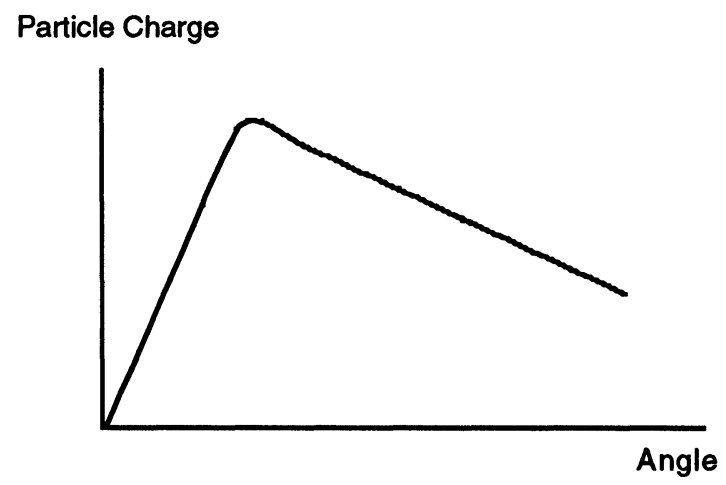

Fig. 6a Typical particle charge profile on the roll

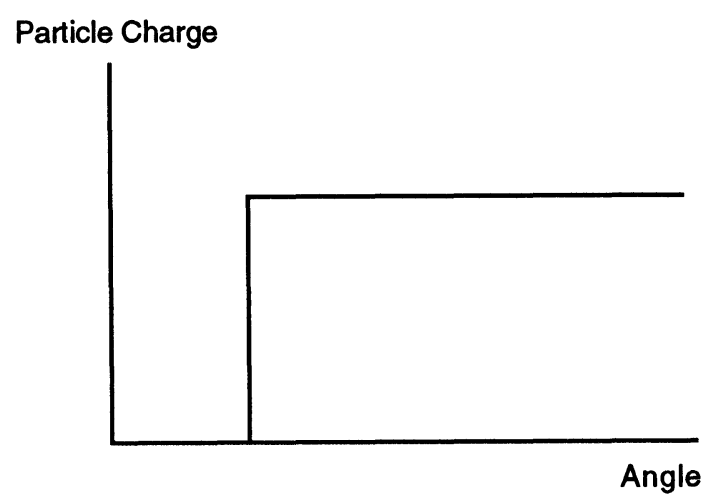

Fig. 6b Simplified model of particle charge on the roll

For the purposes of this work, a simplified charge model was used, in which the corona field commenced at a roll angle of 15 degrees from the vertical, and the charge on the particle does not decay, corresponding to it being a non-conductor mineral such as zircon.

This simplified model is shown diagrammatically in Fig. 6b. This model results in a constant image force $\mathrm{F}_{\mathrm{I}}$ once the particle has moved within the corona field. The electrostatic pinning force $F_{P}$ should decline as the particle is carried away from the influence of the static electrode and the field strength $E$ declines, despite the charge $q$ remaining constant (eq. (5)). In the simplified model the field strength $E$ 
is also assumed constant. This approach will clearly over-estimate the influence of electrical forces on the separation process.

The image and electrostatic forces calculated using eqs. (4) and (5) were included in the radial force balance to determine the nett radial force and hence the frictional force, and the equations solved numerically, as before.

The coefficient of friction was estimated for $160 \mu \mathrm{m}$ zircon particles by placing them on a smooth inclined steel plate and increasing the angle of tilt until they just began to slip or roll. The critical angle was found to be 18 degrees, corresponding to a friction coefficient $\mu_{\mathrm{d}}$ of 0.32 [9].

The numerical results are shown in Fig. 7, for three different zircon particle sizes: 100,160 and $250 \mu \mathrm{m}$. It can be seen that even after including the additional forces due to corona effects, particle speed is still less than $250 \mathrm{rpm}$, even after travelling 90 degrees around the roll, despite the exaggeration of the influence of the electrical forces on the radial force balance by the simple model used.

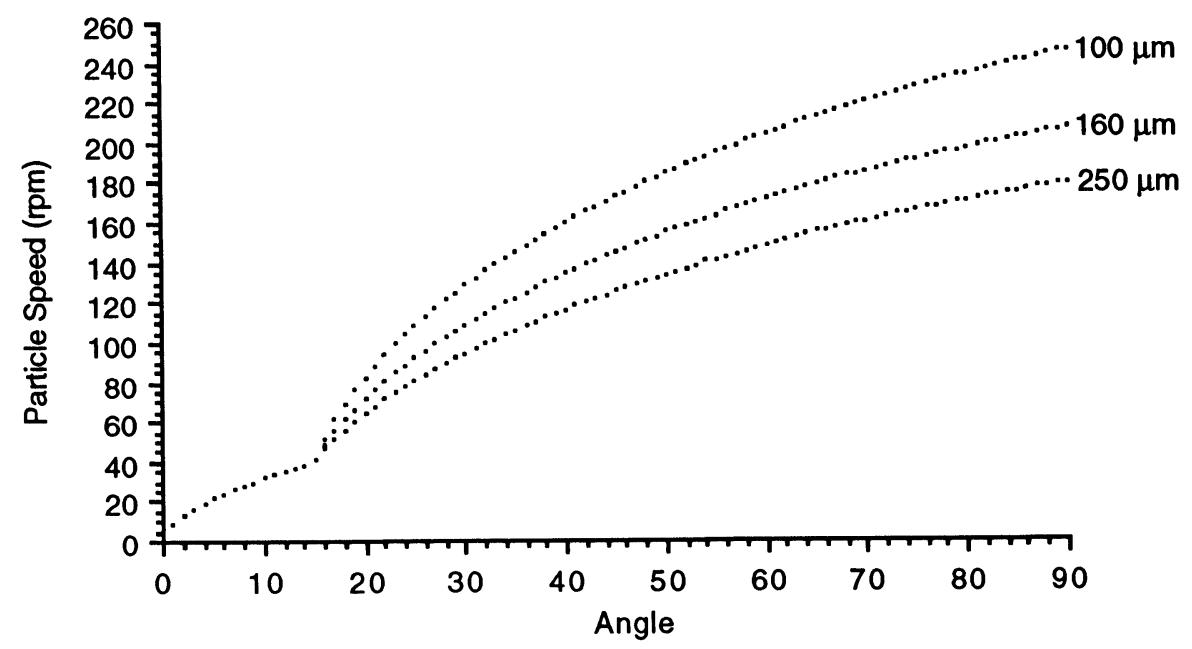

Fig. 7 Particle speed on the roll - numerical solution (friction coefficient 0.32 ) 
Particle speed will depend to some extent on the value of friction coefficient used. Fig. 8 shows the sensitivity of the solution to different values of the friction coefficient for $160 \mu \mu$ zircon particles. It can be seen that particle speed is not particularly sensitive to the value of friction coefficient used in the calculations, and that even with a quite large value the particles still do not approach typical roll speeds.

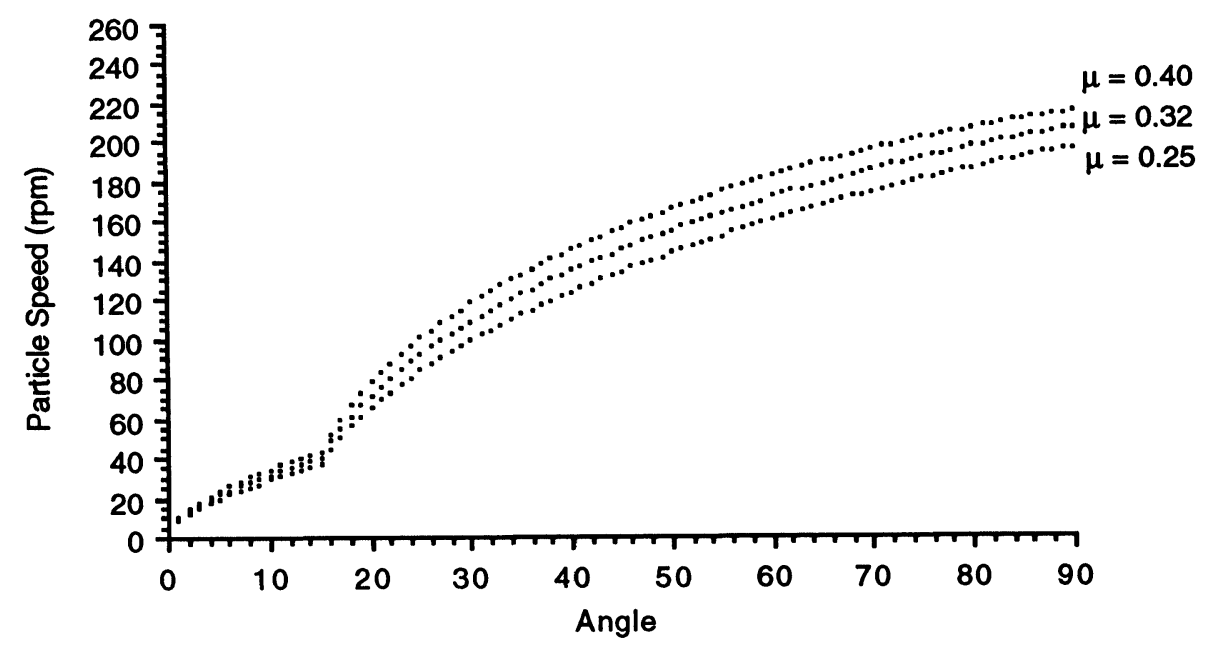

Fig. 8 The effect of varying friction coefficient on particle speed numerical solution (particle size $160 \mu \mathrm{m}$ )

The theoretical analysis above clearly suggests that particles on the high tension roll do not instantaneously reach roll speed as has previously been assumed. However, the simple model of the electrical forces used and the uncertainties associated with measurement of the friction coefficient means that experimental confirmation is necessary. 


\section{EXPERIMENTAL MEASUREMENT OF PARTICLE VELOCITY ON THE ROLL}

Measurement of particle velocity on the HTR roll is difficult and in the literature does not appear to have been attempted, probably due to the widely held assumption that no particle slippage occurs.

Velocity measurement in the present work was carried out by taking a series of photographs of particles moving on the roll. By careful choice of shutter speed and lighting, it was possible to measure particle velocity from the trail length produced in a photograph with a known exposure time. A stationary tape measure suspended next to the roll surface was used to determine position and scaling within the photographs.

The experiment was performed using $+150-180 \mu \mathrm{m}$ zircon particles, a roll speed of $275 \mathrm{rpm}$ and a camera shutter speed of $1 / 500$ th of a second. To avoid excessive overlapping of particle trails in the photographs, extremely low feed rates were employed, achieved by hand-feeding at the rate of about 100 grams per minute. A Mineral Deposits laboratory HTR machine was used, configured as shown in Table 1.

Table 1 Laboratory HTR configuration

$\begin{array}{ll}\text { Roll diameter } & 236 \mathrm{~mm} \\ \text { Roll speed } & 275 \mathrm{rpm} \\ \text { Electrode voltage } & 25 \mathrm{kV} \\ \text { Corona electrode gap from roll surface } & 55 \mathrm{~mm} \\ \text { Static electrode gap from roll surface } & 59 \mathrm{~mm} \\ \text { Corona electrode angle } & 45^{\circ} \text { from vertical } \\ & \text { through roll centre } \\ \text { Static electrode angle } & 58^{0} \text { from vertical } \\ & \text { through roll centre }\end{array}$




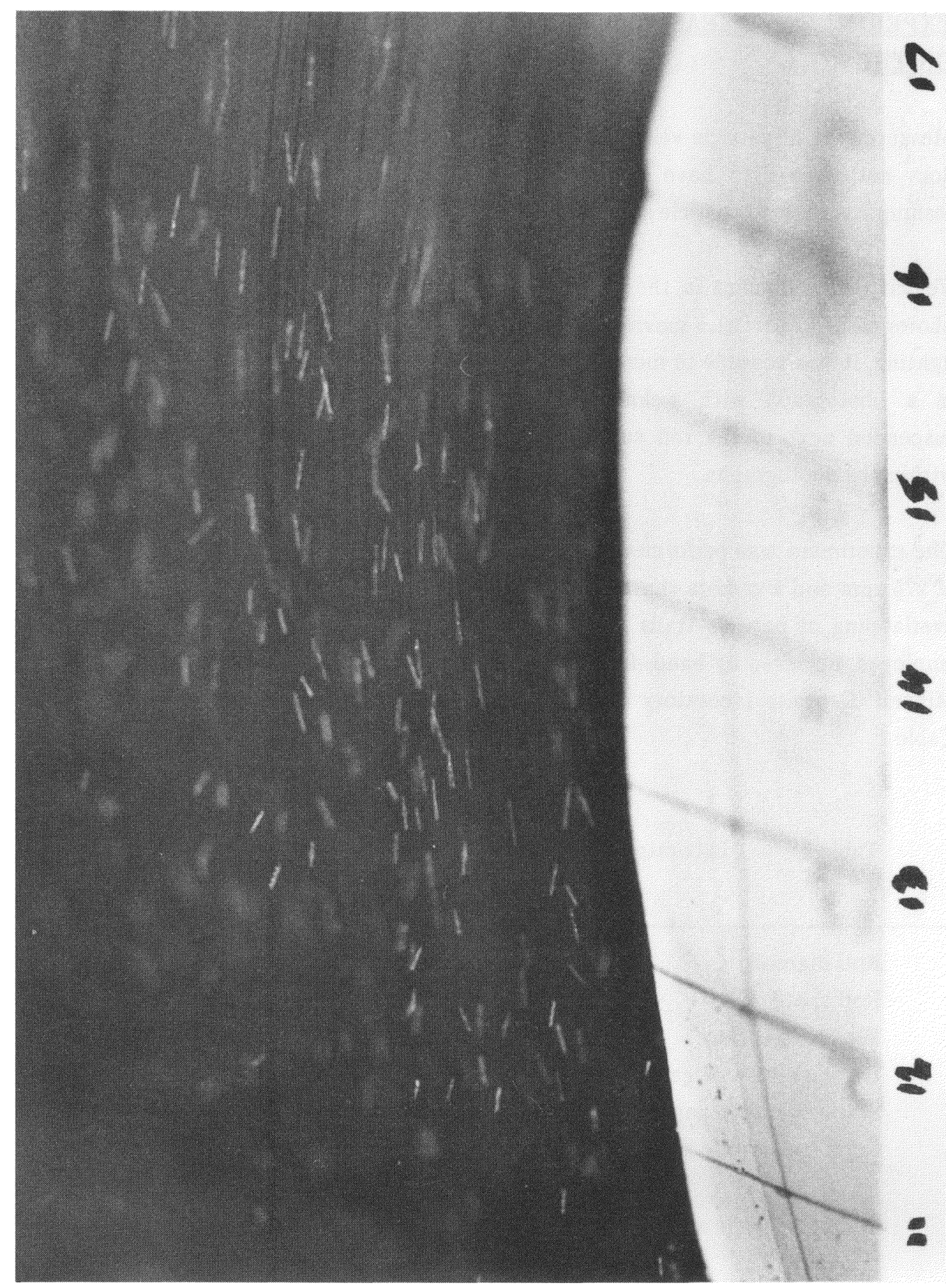

Fig. 9

Trails of zircon particles in the HTR separator 
The photographs were analysed by measuring particle trail lengths at different roll positions (steps $1 \mathrm{~cm}$ on the roll circumference) and scaling to give the true velocity. Fig. 9 is an example of one of the particle trail photographs. Four particle trails were measured at each position and Fig. 10 shows the mean measured particle velocities as a function of position on the roll superimposed on the calculated velocity curve for comparison.

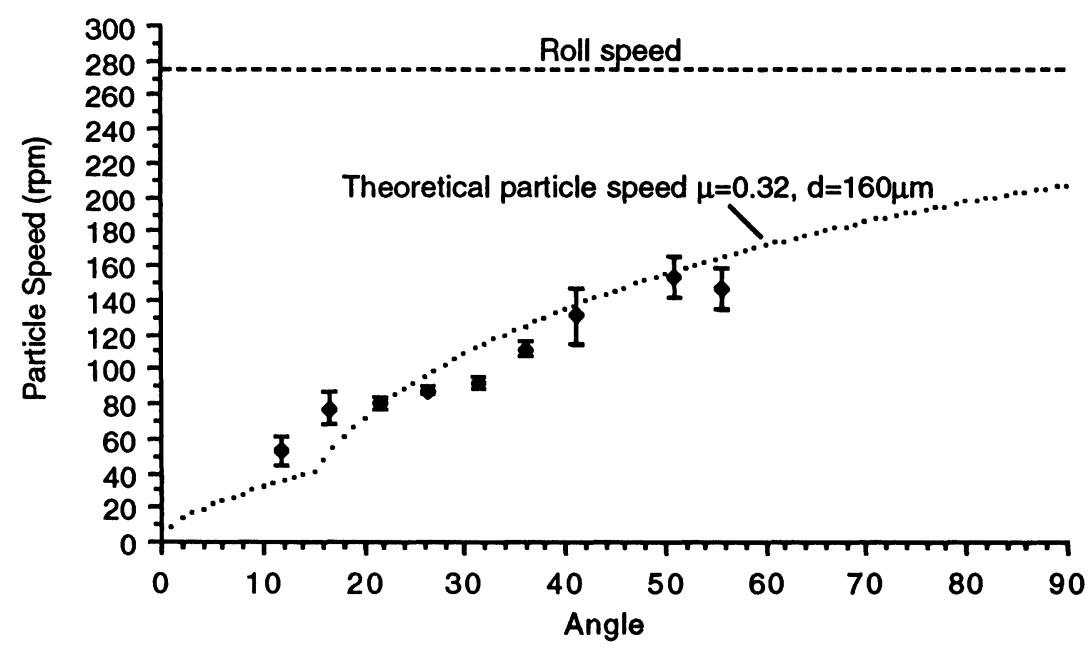

Fig. 10 Measured zircon particle speeds (diameter roll $0.236 \mathrm{~m}$ )

Not only do the results shown in Fig. 10 confirm the existence of particle slippage on the roll surface, but the agreement with the theoretical particle velocity calculations is encouraging considering the simplified model of the electrical forces used.

Examination of the particle trails in Fig. 9 shows that they exhibit periodic changes in brightness along the length of the trail. The brightness changes are evidence that the particles are rolling, rather than being pinned firmly to the roll surface, which in itself is further evidence that the particles are not travelling at the roll speed. The period of the fluctuations appears to be about 0.5 milliseconds. 


\section{CONCLUSIONS}

The work described in this paper forms part of a larger programme of research into electrostatic separation of minerals sands currently being carried out at the Julius Kruttschnitt Mineral Research Centre. The objective of the work is the progressive refinement of existing models of the high tension roll separator to reduce the degree of empiricism and enhance their general validity. From a theoretical standpoint, it has been shown that it is likely that particles on the rolls of industrial scale separators do not always reach roll speed and thus the assumption that particle speed and roll speed are the same (after brief initial acceleration of the particle) is not applicable.

Experimental evidence for $160 \mu \mathrm{m}$ zircon particles at low feed rates support the theoretical analysis. This finding has important implications for the development of robust models of these separators. If particles are travelling at less than the roll speed, the radial force balance will be significantly altered and thus prediction of particle speed becomes critical in defining the point at which a particle leaves the roll However, the effect of larger particle feed rates on the roll remains to be determined. The current HTR model in JKSimSand will be revised to incorporate particle speed effects and validated with data from HTR machines at a number of Australian mineral sand plants.

\section{REFERENCES}

[1] R.G. Mora: Study of electrical concentration of minerals. MSc. Thesis (unpublished), Massachusetts Institute of Technology, Cambridge, Mass., USA (1958)

[2] C. Nilkuha: The examination of some aspects of high tension separation of minerals. MEngSci Thesis (unpublished), University of Melbourne, Australia (1959)

[3] R.D. Morrison: Mathematical modelling of high tension roll separation. PhD Thesis (unpublished), University of Queensland, Australia (1977)

[4] A.D. Dance: Modelling the electrostatic separation of mineral sands. PhD Thesis (unpublished), University of Queensland, Australia (1992) 
[5] A.D. Dance, R.D. Morrison, T. Kojovic: Development of electrostatic separation models for the minerals sands industry. Proc. AusIMM Extractive Metallurgy Conf., Perth (1991), 13

[6] E.G. Kelly, D.J. Spottiswood: The theory of electrostatics separations: A review. Parts 1 to 3. Minerals Eng. 2 (1989)

[7] I. Cakir, A.J.W. Rozelaar, I.S. Wells: A preliminary analysis of the mechanical forces acting on a particle on the rotor of an electrostatic separator. In: Proc. XII Int. Miner.Proc. Congress, Sao Paulo, Brazil (1977), 47

[8] A.W. Stradling: Mathematical models of dry high intensity magnetic separators. PhD Thesis (unpublished), University of Queensland, Australia (1991)

[9] D. Edward: A study of electrostatic separation models and their application. MSc. Thesis (unpublished), University of Queensland, Australia (1994)

\section{ACKNOWLEDGMENTS}

This work was financially supported by the following companies: Aberfoyle, Amdel, Cable Sands, Mineral Deposits, Tiwest, RGC Mineral Sands, Westralian Sands, through Australian Mineral Industry Research Association (AMIRA) project P255A "Advances in Mineral Sands Beneficiation". D. Edward received an Australian Postgraduate Award (Industry).

Darren J. Edward graduated in 1991 with a B. Eng. degree in Mineral Processing from the University of Queensland. He completed his M. Eng. degree at the Julius Kruttschnitt Mineral Research Centre in 1994, his research topic being electrostatic separation. He is currently a consultant in coal preparation with Clarkson \& Associates, Brisbane.

Peter Holtham graduated in 1973 from the University of Leeds with a B.Sc. degree in Applied Mineral Science. He obtained his Ph.D. degree from the University of New South Wales in 1990 and is currently a Senior Research Fellow at the Julius Kruttschnitt Mineral Research Centre.

Toni Kojovic: for biography see Magn. Electr. Sep. 5 (1994)

Keywords: High tension roll separator, modelling, particle motion 